See discussions, stats, and author profiles for this publication at: https://www.researchgate.net/publication/316047544

\title{
Wing Polymorphism and Trypanosoma cruzi Infection in Wild, Peridomestic, and Domestic Collections of Mepraia spinolai (Hemiptera: Reduviidae) From Chile
}

Article · March 2017

DOI: $10.1093 / \mathrm{jme} / \mathrm{tj} \times 061$

CITATIONS

7 authors, including:

Daniel Frias

Universidad Metropolitana de Ciencias de la Educación

46 PUBLICATIONS 381 CITATIONS

SEE PROFILE

Danila Blanco de Carvalho

10 PUBLICATIONS 69 CITATIONS

SEE PROFILE

Some of the authors of this publication are also working on these related projects:
READS

136

Christian R González

Universidad Metropolitana de Ciencias de la Educación 102 PUBLICATIONS 311 CITATIONS

SEE PROFILE

Jader de Oliveira

São Paulo State University

66 PUBLICATIONS 250 CITATIONS

SEE PROFILE

Project Importancia de Canis familiaris, como centinela y reservorio de la enfermedad de Chagas View project

Triatomines genetics View project 


\title{
Wing Polymorphism and Trypanosoma cruzi Infection in Wild, Peridomestic, and Domestic Collections of Mepraia spinolai (Hemiptera: Reduviidae) From Chile
}

\author{
Daniel Frías-Lasserre, ${ }^{1,2}$ Christian R. González, ${ }^{1,3}$ Carolina Reyes Valenzuela, ${ }^{3}$ Danila \\ Blanco de Carvalho, ${ }^{4}$ Jader Oliveira, ${ }^{4}$ Mauricio Canals, ${ }^{5,6}$ and Joao Aristeu da Rosa ${ }^{4}$
}

${ }^{1}$ Instituto de Entomología, Universidad Metropolitana de Ciencias de la Educación, Av, José Pedro Alessandri 774, Santiago, Chile 7760197 (daniel.frias@umce.cl; christian.gonzalez@umce.cl), ${ }^{2}$ Corresponding author, e-mail: daniel.frias@umce.cl, ${ }^{3}$ Laboratorio Entomología, Sección Parasitología, Instituto de Salud Pública de Chile (creyes@ispch.cl), ${ }^{4}$ Departamento de Ciências Biologicas, Faculdade de Ciências Farmacêuticas, Universidade Estadual Paulista, Rodovia Araraquara- Jaú km 1, 14 801-902, Araraquara, SP, Brasil (danilablanco@gmail.com; jdr.oliveira@hotmail.com; rosaja@fcfar.unesp.br), ${ }^{5}$ Programa de Salud Ambiental, Escuela de Salud Pública, Facultad de Medicina, Universidad de Chile; Departamento de Medicina, Facultad de Medicina, Universidad de Chile. Independencia 939, Santiago, Zip code 8380453. Web:www.mauriciocanals.cl. (mcanals@uchile.cl), and ${ }^{6}$ Departamento de Medicina, Facultad de Medicina, Universidad de Chile

Subject Editor: David Florin

Received 6 December 2016; Editorial decision 28 February 2017

\begin{abstract}
Mepraia spinolai (Porter) is a vector of Trypanosoma cruzi that causes Chagas disease. Females are always wingless, but males may be winged or wingless. We determined by PCR the infection percentage with $T$. cruzi of $M$. spinolai adults and nymphs in domestic, peridomestic, and wild collections, in different regions of Chile. In all regions, winged males were more abundant than females and wingless males. Winged males collected inside houses were less parasitized than were those from peridomestic and wild environments. Although winged males of $M$. spinolai have comparatively low levels of infection, this segment may still represent the greatest vector threat in this species for transmission of $T$. cruzi to humans and other vertebrates in domestic, wild, and peridomestic habitats. Winged males represent the dispersive form of this species that invades human dwellings. Feeding deprivation resulting from the time required to find a food source and to search for reproductive females could explain the lower infection rates (negatives) of winged males collected from inside houses in comparison with winged males collected from peridomestic and wild habitats.
\end{abstract}

Key words: Mepraia spinolai, Chagas disease, human dwelling, wing polymorphism

In Chile, Trypanosoma cruzi transmission from vectors to humans occurs mainly in rural and suburban areas of the desert and also in semiarid and Mediterranean environments, between latitudes $18^{\circ}$ $30^{\prime} \mathrm{S}$ and $34^{\circ} 36^{\prime} \mathrm{S}$ (Apt and Reyes 1986, González and Reyes 2015). The principal vector of T. cruzi in domestic and peridomestic environments in Chile is Triatoma infestans (Klug). Although control programs have eliminated T. infestans from large urban areas of Chile (Bacigalupo et al. 2012), new sylvatic foci of this species have been reported in central Chile, in Calera de Tango in the southern zone of the Santiago Metropolitan region, in rock piles in the Valparaíso region, and in the Atacama region (Bacigalupo et al. 2006, Bacigalupo et al. 2010, Bacigalupo et al. 2015). The sylvatic transmission cycle of Chagas disease in Chile includes wild mammals, humans, and vector species within the genus Mepraia (Hemiptera: Reduviidae: Triatominae) and also T. infestans (Coronado et al. 2009, Botto-Mahan et al. 2015, Campos-Soto and
Torres-Pérez 2015). There are three species in this genus: Mepraia gajardoi Frías, Henry and González; M. spinolai (Porter); and M. parapatrica Frías-Lasserre (Lent et al. 1994, Frías et al. 1998, Frías-Lasserre 2010, Botto-Mahan et al. 2015, González et al. 2015, Campos-Soto et al. 2016). Recently published information on T. cruzi infecting M. gajardoi indicated a $5.8-11.8 \%$ of field-collected specimens were infected (Botto-Mahan et al. 2008, González et al. 2015). The distribution of M. gajardoi close to human settlements, such as fishing coves, constitutes a risk to human populations. The risk is higher in M. spinolai because infection rates with T. cruzi can reach 46-61\% in some areas of central Chile (Frias et al 1995, BottoMahan et al. 2015). The percent of infected specimens with T. cruzi in M. spinolai could change in relation to the abundance and richness of mammals host in the area (Coronado et al. 2009). There are currently no records of $T$. cruzi infection rates in M. parapatrica populations because the species has not yet been studied in this respect. 
Currently, the distribution of M. spinolai ranges from the Atacama region to Metropolitan region $\left(26^{\circ}\right.$ to $33^{\circ}$ S; Frías-Lasserre 2010). Considering that T. infestans has been eradicated from urban environments (Bacigalupo et al. 2012), M. spinolai may be a vector in these habitats. Recently, M. spinolai and T. infestans have been collected in wild environments associated with small mammals (Bacigalupo et al. 2010). M. spinolai is commonly found associated with small rodents and rabbits in rocky sylvatic ecotopes, in henhouses and goat corrals in peridomestic ecotopes (Campos et al. 2007), and, occasionally, has been reported in homes (Canals et al. 1999). Unlike other triatomines, this species is diurnal, exhibiting aggressive feeding behavior on the host during the day (Gajardo-Tobar, 1938, Gajardo-Tobar 1960, Apt and Reyes 1986, Frías et al. 1987, Canals et al. 1997, Canals et al. 1999) although it is also active at night (Canals et al. 2001). The first evidence that M. spinolai may be vector of T. cruzi to humans and other vertebrates comes from Gajardo-Tobar's (1953), where the species was found to have a wide range of infection with T. cruzi. Besides, in several places from Chile he observed how the insects sucked blood from humans and other wild and domesticated mammals and also lizards and snakes. The presence of human blood and other vertebrates in the intestinal content of the insects, was detected by lapine antiserum antiseric antigens; in decreasing order, the prevalence of feeding sources were rabbits, dogs, goats, rodents, humans, and birds (hens); reptilian blood was not detected in the intestinal content of any insects collected (Canals et al. 2001). Other direct evidence that T. cruzi infects the blood of native mammals comes from Southern blot analysis and hybridization with the probe of kDNA (PCR; Botto-Mahan et al. 2010).

Adult females and males of most Triatominae are winged (Lent and Wygodzinsky 1979), but the genus Mepraia, which is endemic to Chile, is characterized by wing polymorphism. Females are always wingless, but males may be winged or wingless (Mazza et al.1940, Gajardo-Tobar 1953, Gajardo-Tobar 1960, Lent and Jurberg 1967, Lent et al. 1994). The geographic distributions of winged and wingless males vary among different habitats, and possible relationships between T. cruzi infection and alary polymorphism of M. spinolai, have not been investigated. We hypothesize that there would be differences in infection rates of T. cruzi among populations of M. spinolai that inhabit wild, peridomestic and domestic conditions. We also speculate that the alary polymorphism might be related to the type of habitat and intensity of infection by T. cruzi. At present, it is unknown which instar of M. spinolai is most common in human dwellings. We postulate that females and wingless males, and also nymphs, would have a lower dispersive capacity than winged males. In that case, we should find more winged males within human habitations. This increased dispersive capacity of winged males might be reflected in lower infection rates by $T$. cruzi compared with females and wingless males.

In the present study, we report T. cruzi infection rates of nymphs, females, and males of M. spinolai in wild, peridomestic, and domestic environments in different regions of Chile. We also describe the relationship between parasitism by $T$. cruzi and wing polymorphism in males of M. spinolai. The objectives of this study were 1) to determine by PCR the number and proportion of adults and nymphs of M. spinolai infected with T. cruzi in wild, peridomestic, and domestic environments, in different regions of Chile; and 2) to determine whether their alar polymorphism influences their invasion of domestic environments.

\section{Materials and Methods}

\section{Places and Microhabitats Where Specimens Were Collected}

A total of 833 adults and nymphs of M. spinolai from Atacama, Coquimbo, and Valparaíso Regions were collected. In all the localities where the specimens were collected, M. spinolai was the exclusive species collected and not the other two species of Mepraia (Frías-Lasserre 2010; Fig. 1). Samples were taken during the years 2012 to 2015. Specimens were obtained from three different places: 1) inside human habitations, both in urban and rural rooms; 2) in the peridomestic habitats, particularly in corrals of domestic animals such as goats, rabbits, hens, horses, and pigs; and 3) in wild places, such as piles of stones where wild small rodents live. All the specimens studied were collected by the Institute of Public Health (ISP) under the auspices of the Ministry of Health of Chile. Triatomines tested were collected manually from 1200 to 1900 hours by three people, who sampled along various points in a stony area, near human dwellings including animal pens. All of the captured specimens were placed into individual plastic containers. Other specimens were sent to us by health professionals belonging to Regional Ministerial Secretariats of Chile (SEREMIS) of the different regions. Each specimen was tested for infection with T. cruzi. We tabulated the number of infected and uninfected individuals in domestic, peridometic, and wild habitats and then calculated whether the differences were statistically significant by chi-square $\left(\chi^{2}\right)$.

\section{Taxonomic Identification of Captured Specimens}

Identification of captured specimens was based on the work of Gajardo-Tobar (1953), Lent and Jurberg (1967), Frías et al. (1987), Jurberg et al. (2002), Lent et al. (1994), Frías et al. (1998), Frías and Atria (1998), and Frías-Lasserre (2010). Winged males are easily distinguished from females and wingless males. However, some wingless males could be mistaken for females and, in these cases, they were differentiated by their genitalia.

\section{Detection of T. cruzi DNA in M. spinolai by PCR}

The detection of T. cruzi DNA in the insect specimens was conducted using PCR techniques in homogenates of the distal portion of the intestine. DNA extraction from the intestinal tract was performed in a Maxwell 16MDx instrument (Promega, Madison, WI), using a Maxwell 16 Tissue DNA purification kit (Promega). Conventional PCR was performed using an Applied Biosystems 2720 thermal cycler (Applied Biosystems, Carlsbad, CA). The PCR master mix consisted of $1 \times$ reaction buffer, $0.25 \mathrm{mM}$ deoxynucleotide triphosphate solution (dNTP), $3 \mathrm{mM} \mathrm{MgCl}_{2}$ solution, $0.6 \mathrm{U}$ Taq Platinum (Invitrogen, Carlsbad, CA), $2.5 \mu \mathrm{m}$ kDNA specific primers $121\left(5^{\prime}\right.$-AAATAATGTACGGGGGAGATGCATGA-3') and $122\left(5^{\prime}-\right.$ GGTTCGATTGGGGTTGGTGTAATATA-3'), $5 \mu$ l of template DNA, and a quantity of water sufficient for a final volume of $30 \mu \mathrm{l}$. Cycling parameters were one 5 -min denaturation step at $94^{\circ} \mathrm{C}$; 33 cycles of $1 \mathrm{~min}$ at $94^{\circ} \mathrm{C}, 1 \mathrm{~min}$ at $65^{\circ} \mathrm{C}$, and $1 \mathrm{~min}$ at $72{ }^{\circ} \mathrm{C}$; and one final extension step of $10 \mathrm{~min}$ at $72^{\circ} \mathrm{C}$. PCR products of $330 \mathrm{bp}$ were analyzed in $1 \%$ agarose gels stained with GelRed Nucleic Acid Gel Stain (Biotium, Hayward, CA). The methodology used follows the proposal by Schijman et al. (2011) and Enriquez et al. (2013).

\section{Results}

\section{Numbers of Each Instar of M. spinolai Collected During the Years 2012-2015}

Table 1 shows the number of individuals collected in each region in wild, peridomestic, and domestic habitats. In all regions, infected specimens exceeded $26 \%$. The percentage of infected individuals is lower in domestic than the peridomestic and wild habitats. In the different regions and habitats, the number of winged males was greater than wingless males or females. Nymphs were most 

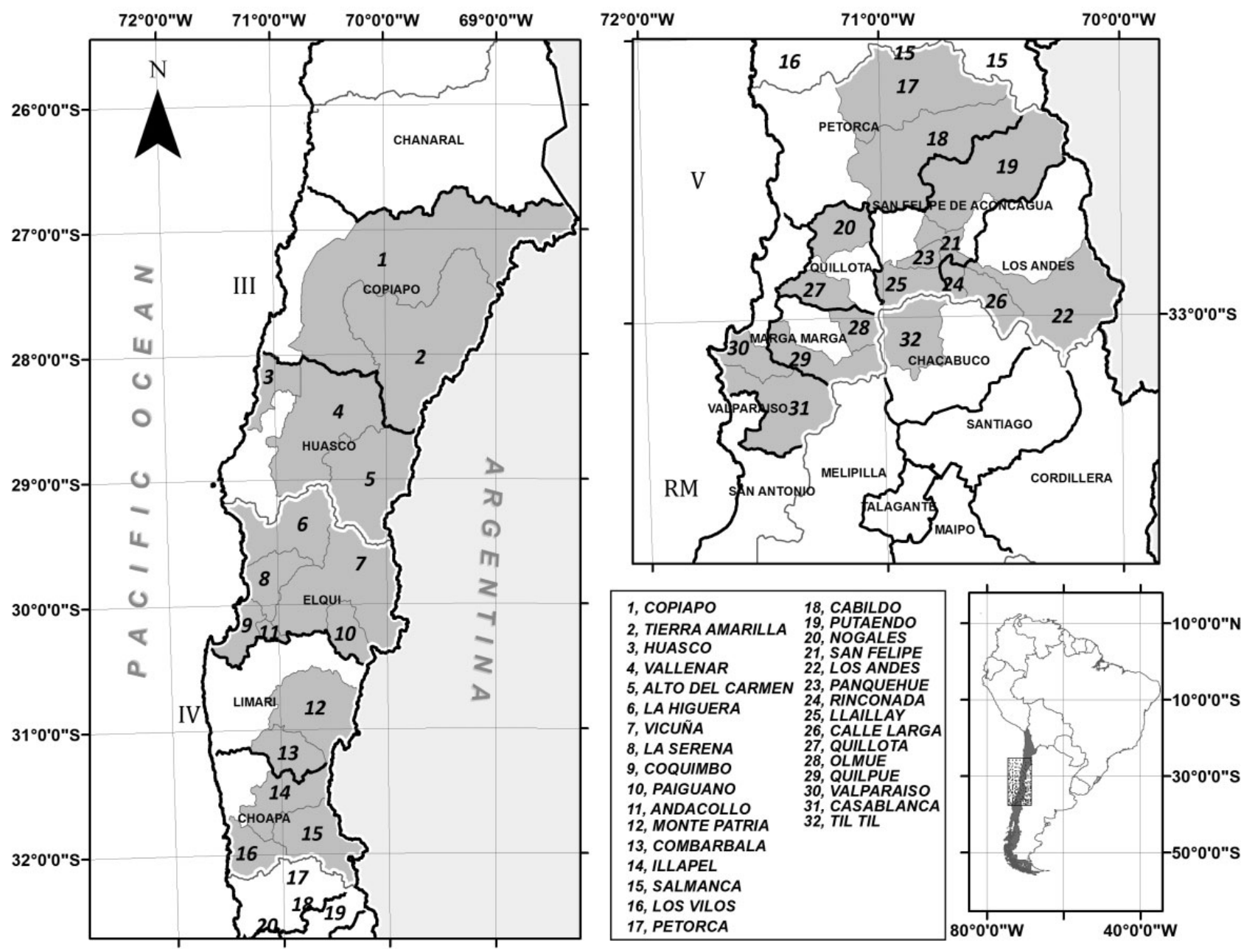

Fig. 1. Map of Chile showing the regions, provinces, and communes where the specimens were collected. The numbers on the map represent the communes of each regions where the specimens were captured. Roman numerals correspond to regions of Atacama (III), Coquimbo (IV), Valparaiso (V), and Metropolitan (RM).

abundant in the wild habitat, particularly in Coquimbo and Valparaíso. More winged males were recorded in the domestic environment $(n=329)$ than in the peridomestic $(n=73)$ and wild $(n=111)$ areas. In all regions, the number of infected winged males was less in the domestic habitat than in the peridomestic and wild habitats. Among all individuals caught during 2012 to 2015, the proportion of infected (positive) individuals was lower in domestic (0.31) than in peridomestic (0.63) and in wild environments $(0.55)$. The proportion of infected winged males was significantly lower in domestic environments than in the peridomestic and wild environments $\left(\chi^{2}=20.58, P<0.0001 ; \chi^{2}=46.46, P<0.0001\right.$, respectively). In peridomestic and wild environments, the proportion of infected winged males was higher than in domestic environments, but the differences were not significant at the 0.05 level of significance $\left(\chi^{2}=5.46, P=0.0194\right.$; Table 2$)$. No data were obtained during 2015 for wild and peridomestic areas, and the analysis was repeated excluding 2015, with similar results (human dwellings vs. peridomestic environments, $\chi^{2}=16.00, P<0.0001$; domestic vs. wild, $\chi^{2}=39.39, P<0.0001$; and peridomestic vs. wild, $\chi^{2}=4.80$, $P=0.03)$.

The proportion of infected males collected in domestic environments was significantly lower than the proportion of infected males collected in wild + peridomestic habitats combined $\left(\chi^{2}\right.$ test, Table 3$)$

\section{Discussion}

Considering that M. spinolai has been shown to be a vector of T. cruzi (Canals et al. 2001, Botto-Mahan et al. 2010), the risk of being infested with T. cruzi is high, as in all regions infected specimens were detected at levels that exceeded $26 \%$. The risk, in each region, increases in peridomestic and wild environments, where the percentages of infection with $T$. cruzi increases. The risk of transmitting T. cruzi by winged males in all regions studied is high as revealed by vector infection prevalence of $79 \%$ in wild environments, $63 \%$ in peridomestic habitats, and $30 \%$ in domestic habitats. However, we could argue that the diurnal behavior of M. spinolai implies less chance of sustained blood feeding and thus transmission of T. cruzi via defecation.

We collected very few nymphs $(0.9 \%)$, females (3.28), and wingless males (0.9) inside houses, but many winged males (39.9). This suggests that the winged males are the dispersive form of M. spinolai, as has been described in other insects where there is wing polymorphism (Dixon et al. 1993).

Inside houses, the proportion of uninfected (negative) winged males was greater than in peridomestic and domestic habitats. Feeding deprivation due to dispersion could explain the low infection rate of winged males collected inside houses in comparison with winged males in peridomestic and wild habitats. Even with this 
Table 1. Number of M. spinolai specimens collected by environment and location

\begin{tabular}{lcccc}
\hline $\begin{array}{l}\text { No. of individuals and } \\
\text { type of environment }\end{array}$ & Atacama & Coquimbo & Valparaíso & Total \\
\hline $\begin{array}{l}\text { Wild habitat } \\
\text { Winged males }\end{array}$ & $77(63)$ & $17(13)$ & $17(12)$ & $111(88)$ \\
Wingless males & 3 & $1(1)$ & $3(2)$ & $7(3)$ \\
Females & 0 & $7(6)$ & $15(8)$ & $22(14)$ \\
Nymphs & 1 & $109(55)$ & $125(47)$ & $235(102)$ \\
Total & $81(63)$ & $134(75)$ & $160(69)$ & $375(207)$ \\
Peridomestic & & & & \\
Winged males & $34(30)$ & $7(2)$ & $32(15)$ & $73(46)$ \\
Wingless males & 0 & 0 & 2 & 2 \\
Females & 0 & 1 & 0 & 1 \\
Nymphs & 1 & 0 & 1 & 2 \\
Total & $35(30)$ & $8(2)$ & $35(15)$ & $78(46)$ \\
Domestic & & & & \\
Winged males & $107(36)$ & $61(19)$ & $161(45)$ & $329(100)$ \\
Wingless males & 2 & $1(1)$ & $4(1)$ & $7(2)$ \\
Females & $6(1)$ & $7(4)$ & $14(4)$ & $27(9)$ \\
Nymphs & 3 & $4(2)$ & 0 & $7(2)$ \\
Total & $118(37)$ & $73(26)$ & $179(49)$ & $370(113)$ \\
Total & $234(130)$ & $215(103)$ & $374(133)$ & $823(366)$ \\
\hline
\end{tabular}

Collection occurred in Atacama, Coquimbo, and Valparaíso regions of Chile during the years 2012, 2013, 2014, and 2015 (ISP data). The number of specimens infected with T. cruzi is indicated in parenthesis.

discrepancy, we believe that winged males are probably the most important vectors of T. cruzi inside houses. Food supply is an important biological factor that modulates interactions between $T$. cruzi and its insect vector, together with gut flora and other intestinal components, the strain of T. cruzi, and the insect's physiology (Garcia et al. 2010). In a regularly fed infected triatomine, T. cruzi amplify as epimastigotes, which later differentiate to trypomastigotes that are eliminated by the feces (García et al. 2010). Feeding affects parasite density and insect molting, and also changes the epimastigote/trypomastigote ratio in the rectum of other Triatominae, e.g., T. infestans (Kollien and Schaub 1998). The rectal parasite density increases until several weeks postinfection with regular bloodmeals, reaching maximal values of several million parasites. The parasite density is strongly influenced by very long starvation up to $20 \mathrm{wk}$. So, starvation is inversely correlated with the number of parasites in the vector (Schaub and Böker 1986, Vargas and Zeledón 1985). Egaña et al. (2014) determined T. cruzi infection in M. gajardoi and M. spinolai by assessing infection rates of insects from natural conditions and reassessing them after feeding in the laboratory. One group of M. spinolai seemed to be completely uninfected, but after the first and second feedings, with noninfected blood, $62 \%$ and $59 \%$ were positive, respectively, because the nutrients within led to the proliferation of previously undetectable T. cruzi (Egaña et al. 2014), similar to levels we measured in wild and peridomestic environments. These observations could explain the low infection rate of the winged males found inside houses. Possibly, dispersion, from wild to domestic environments, increases their starvation and the rectal parasite density decreases when they enter houses. Insects collected inside houses had blood in their digestive tract, but whether that blood came from a domestic infestation during which the bugs were feeding on noninfected or infected inhabitants is not known. In addition, we do not know if that blood was from other vertebrates other than humans. Since the detection of T. cruzi DNA in the insect specimens was conducted using PCR
Table 2. Comparison by $\chi^{2}$ test of the number of $T$. cruzi positive and negative winged males of $M$. spinolai collected from domestic, peridomestic, and wild environments

\begin{tabular}{lccc}
\hline Environments & $\begin{array}{c}\text { No. of positive } \\
\text { winged males }\end{array}$ & $\begin{array}{l}\text { No. of negative } \\
\text { winged males }\end{array}$ & $\chi^{2}(P)$ \\
\hline Domestic & 30 & 70 & \\
Peridomestic & 63 & 37 & $20.58(<0.0001)$ \\
Domestic & 30 & 70 & \\
Wild & 79 & 21 & $46.46(<0.0001)$ \\
Peridomestic & 63 & 37 & \\
Wild & 79 & 21 & $5.46(>0.0194)$ \\
\hline
\end{tabular}

Table 3. Comparison by $\chi^{2}$ between the number of $T$. cruzi-infected male $M$. spinolai collected inside domestic environments with the number of infected males collected in wild + peridomestic habitats

\begin{tabular}{lccc}
\hline Environments & \multicolumn{3}{c}{ Regions } \\
\cline { 2 - 4 } & Atacama & Coquimbo & Valparaíso \\
\hline Wild + Peridomestic & 85 & 63 & 55 \\
Domestic & 34 & 31 & 29 \\
$X^{2}$ & 51.87 & 19.29 & 12.82 \\
$P$ & $<0.0001$ & $<0.0001$ & $<0.0001$
\end{tabular}

techniques in homogenates of the distal portion of the intestine, we assume that the DNA comes from trypomastigotes that develops in the rectum and is eliminated by the feces (García et al. 2010). Also, since PCR is not a quantitative method, it would be necessary to measure the proportion of human red blood cells and that of other wild and domesticated mammals in the vectors infected with T. cruzi from each microhabitat following the method of Canals et al. 2001.

It is interesting that large numbers of winged males blood-fed were recorded inside human habitations because in wild and in peridomestic environments winged males are less frequent or have similar occurrence than wingless males (Frías-Lasserre unpublished data). One explanation could be that the eradication of T. infestans in urban areas has facilitated the entry of M. spinolai to domestic environments. Testing in the laboratory has indicated that the presence of T. infestans interferes negatively in the distribution of M. spinolai in the experimental system (Frías et al. 1995). Experiment modeling (Kingsolver and Koehl 1985, Gillott 2005) suggests that wings also function as thermoregulatory structures to increase body temperature by absorbing radiation and allowing migration (Douglas 1981). Therefore, the wings of winged males of $M$. spinolai might conserve body heat and induce searching via walking for refuges in domestic habitats, where the temperature in summer is lower than in wild habitats. Insects with longer wings should be abundant in cool climates, while those without wings or very short wings should be principally present in warm environments. Field observations and laboratory tests have shown that winged males do not fly and do not spread their wings; dispersion is by walking (Frias-Lasserre unpublished observations). If the absence of wings favors thermoregulation through the liberation of body heat, then the wingless males and females should be found frequently in hot microhabitats. Current temperatures in summer 2017 in different microhabitats show that, in rural areas, temperatures in human houses are lower than wild and peridomestic habitats. For instance, in Nogales (Valparaíso Region) in the piles of stone, wild habitats of 
M. spinolai, associated to small rodent, the temperature was $36^{\circ} \mathrm{C}$ and inside rural houses $20^{\circ} \mathrm{C}$. In Combarbala (Coquimbo Region) in a goat pen where M. spinolai live the temperature was $30^{\circ} \mathrm{C}$ and inside a rural house $21^{\circ} \mathrm{C}$. Based on our data, we suggest that disruptive selection pressures may be acting on $M$. spinolai males. These pressures could be responsible for the wing polymorphism observed in natural populations of M. spinolai examined in this study.

\section{Acknowledgments}

We thank Christiane Weirauch for his suggestions on the manuscript. We also thank two anonymous reviewers for their comments and suggestions and to Diego Pinto Veas for the elaboration of Figure 1. This work was supported by projects FIF 0214 DIUMCE and Fondecyt 1150514.

\section{References Cited}

Apt, R. M., and H. Reyes. 1986. Aspectos epidemiológicos de la enfermedad de Chagas I. Distribución geográfica, índices de infección en vectores y humanos. Parasitol. al Día. 10: 94-101.

Bacigalupo, A., M.J.A. Segura, C. A. García, C. J. Hidalgo, G. S. Galuppo, and P. E. Cattan. 2006. First finding of Chagas disease vectors associated with wild bushes in the Metropolitan Region of Chile. Rev. Med. Chile. 134: 1230-1236.

Bacigalupo, A., F. Torres-Pérez, V. Segovia, A. García, J. P. Correa, L. Moreno, P. Arroyo, and P. E. Cattan. 2010. Sylvatic foci of the Chagas disease vector Triatoma infestans in Chile: description of a new focus and challenges for control programs. Mem. Inst. Oswaldo Cruz, Rio De Janeiro 105: 633-641.

Bacigalupo, A., V. Segovia, A. García, C. Botto-Mahan, S. Ortiz, A. Solari, M. Acuna-Retamar, F. Torres-Pérez, and P. E. Cattan. 2012. Differential pattern of infection of sylvatic nymphs and domiciliary adults of Triatoma infestans with Trypanosoma cruzi Genotypes in Chile. Am. J. Trop. Med. Hyg. 87: 473-480. doi:10.4269/ajtmh.2012.11-0237.

Bacigalupo, A., J. P. Correa, A. García, and P. E. Cattan. 2015. Focos silvestres de Triatoma infestans en Latinoamérica:análisis y perspectivas para Chile. Parasitol. Latinoamericana 64: 27-35.

Botto-Mahan, C., M. Sepúlveda, M. Vidal, M. Acuña-Retamar, S. Ortiz, and A. Solari. 2008. Trypanosoma cruzi infection in the sylvatic kissing bug Mepraia gajardoi from Chilean sothern pacific ocean cost. Acta Trop. 105: 166-169.

Botto-Mahan, C., R. Campos, M. Acuña-Retamar, X. Coronado, P. Cattan, and A. Solari. 2010. Temporal variation of Trypanosoma cruzi in Chile. Vector-Borne Zoonotic Dis. 10: 317-319. doi:10.1089=vbz.2009.0006.

Botto-Mahan, C., J. P. Correa, A. Bacigalupo, R. Campos-Soto, P. E. Cattan, and A. SolarI. 2015. Ecology of the endemic sylvatic triatomines of Chile. Parasitol. Latinoamericana 64: 12-19.

Canals, M., R, Solís, J. Valderas, M. Ehrenfeld, and P. E. Cattan. 1997. Preliminary studies on temperature selection and activity cycles of Triatoma infestans and T. spinolai (Heteroptera: Reduviidae), Chilean vectors of Chagas disease. J. Med. Entomol. 34: 11-17.

Canals, M., R. Solís, C. Tapia, M. Ehrenfeld, and P. Cattan. 1999. Comparison of some behavioral and physiological feeding parameters of Triatoma infestans Klug, 1834 and Mepraia spinolai Porter, 1934, vectors of Chagas disease in Chile. Mem. Inst. Oswaldo Cruz 94: 687-692.

Canals, M., L. Cruzat, M. C. Molina, A. Ferreira, and P. E. Cattan. 2001. Blood host sources of Mepraia spinolai (Heteroptera: Reduviidae), wild vector of Chagas disease in Chile. J. Med. Entomol. 38: 303-307.

Campos, R., C. Botto-Mahan, S. Ortiz, M. Acuña-Retamar, P. E. Cattan, and A. Solari. 2007. Trypanosoma cruzi detection in blood by xenodiagnosis and polymerase chain reaction in the wild rodent Octodon degus. Am. J. Trop. Med. Hyg. 76: 324-326.

Campos-Soto, R., and F. Torres-Pérez. 2015. Biogeografía histórica del vector endémico de la enfermedad de Chagas en Chile (Mepraia). Parasitol. Latinoamericana 64: 20-26.
Campos-Soto, R., F. Panzera, S. Pita, C. Lages, A. Solari, and C. BottoMahan. 2016. Experimental crosses between Mepraia gajardoi and M. spinolai and hybrid chromosome analyses reveal the occurrence of several isolation mechanisms. Infect. Genet. Evol. 45: 205-212.

Coronado, X., M. Rozas, C. Botto-Maham, S. Ortiz, P. Cattan, and A. Solari. 2009. Molecular epidemiology of Chagas disease in the wild transmission cycle: the evaluation in the sylvatic vector Mepraia spinolai from an endemic area of Chile. Am. J. Trop. Med. Hyg. 81: 656-659.

Dixon, A.F.G., S. Horth, and P. Kindlmann. 1993. Migration in insects: Cost and strategies. J. Anim. Ecol. 62: 182-190.

Douglas, M. M. 1981. Thermoregulatory significance of thoracic lobes in the evolution of insect wing. Science 211: 84-86.

Egaña, C., F. Vergara, R. Campos, S. Ortiz, C. Botto-Mahan, and A. Solari. 2014. Trypanosoma cruzi infection in Mepraia gajardoi and Mepraia spinolai: The effect of feeding nymphs from the field. Am. J. Trop. Med. Hyg. 91: 534-536. doi:10.4269/ajtmh.13-0721.

Enriquez, G. F., M. V. Cardinal, M. M. Orozco, A. G. Schijman, and R. E. Gürtler. 2013. Detection of Trypanosoma cruzi infection in naturally-infected dogs and cats using serological, parasitological and molecular methods. Acta Trop. 126: 211-217. doi:10.1016/j.actatropica.2013.03.001.

Frías, D., and J. Atria. 1998. Chromosomal variation, macroevolution and possible parapatric speciation in Mepraia spinolai (Porter)(Hemiptera:Reduviidae). Gen. Mol. Biol. 21: 179-184.

Frías, D., H. Martínez, and A. Wallace. 1987. Some taxonomic features of Triatoma spinolai Porter (Hemiptera: Triatominae). Acta Ent. Chilena 14: 155-170.

Frías, D., A. Solari, C. Gonzales, A. Henry, and A. Alviña. 1995. Índices de infección de Mepraia spinolai con Trypanosoma cruzi, su invasión a ambientes domésticos e interacción con Triatoma infestans. Parasitol al Día 19: 195.

Frías, D. A., A. Henry, and C. R. Gonzalez. 1998. Mepraia gajardoi: a new species of Triatominae (Hemiptera: Reduviidae) from Chile and its comparison with Mepraia spinolai. Rev. Chil. Hist. Nat. 71: 177-188.

Frías-Lasserre, D. 2010. A new species and karyotype variation in the bordering distribution of Mepraia spinolai (Porter) and Mepraia gajardoi Frías et al (Hemiptera: Reduviidae: Triatominae) in Chile and its Parapatric Model of Speciation. Neotrop. Entomol. 39: 572-583.

Gajardo-Tobar, R. 1938. El Schizotrypanum cruzi y sus agentes vectores en Chile. Rev. Chilena Hist. Nat. XI.II: 132-137.

Gajardo-Tobar, R. 1953. Algo más sobre Mepraia spinolai (Porter). Hemiptera, Triatomidae. Rev. Chilena Entomol. 3: 117-125.

Gajardo-Tobar, R. 1960. Anotaciones sobre un insecto de importancia médica. Bol. Hosp. Viña Del Mar. 16: 131-141.

García, E. S., A. Genta, P. Azambuja, and G. A. Schaub. 2010. Interactions between intestinal compounds of triatomines and Trypanosoma cruzi. Trends Parasitol. 26: 499-505.

Gillott, C. 2005. Entomology. 3rd ed. Springer, Netherlands.

González, C. R., C. Reyes, A. Canals, A. Parra, X. Muñoz, and K. Rodríguez. 2015. An entomological and seroepidemiological study of the vectorialtransmission risk of Chagas disease in the coast of northern Chile. Med. Vet. Entomol. 29: 387-392. doi: 10.1111/mve.12131.

González, C. R., and C. Reyes. 2015. Triatoma infestans (Klug) (Hemiptera: Reduviidae: Triatominae) en Chile: antecedentes distribucionales y epidemiológicos. Parasitol Latinoamericana 64: 52-55.

Jurberg, J., H. Lent, R. U. Carcaballo, D. da, S. Rocha, C. Galvao, and D. L. Frías. 2002. Estudo moefologico da genitalia externa masculine de Mepraia gajardoi Frías, Henry and González 1998 (Hemiptera. Reduviidae) com comentarios sobre suas relacoes filogenéticas. Entomol. Vect. 9: 559-577.

Kingsolver, J. G., and M.A.R. Koehl. 1985. Aerodinamics, Thermoregulation, and the evolution of insect wings: Differential scaling and evolutionary change. Evolution 39: 488-504.

Kollien, A. H., and G. A. Schaub. 1998. Development of Trypanosoma cruzi after starvation and feeding of the vector-a review. Tokai J. Exp. Clin. Med. 23: 335-340.

Lent, H., and J. Jurberg. 1967. Algumas informaçoes sobre Triatoma spinolai Porter 1934, com um estudo sobre as genitalia externas (Hemiptera: Reduviidae). Rev. Bras. Biol. 27: 273288. 
Lent, H., J. Jurberg, and C. Galvao. 1994. Revalidacao de genero Mepraia Mazza, Gajardo and Jorg, 1940 (Hemiptera, Reduviidae Triatominae). Mem. Inst. Oswaldo Cruz 89: 347-352.

Mazza, S., R. Gajardo-Tobar, and M. E. Jorg. 1940. Mepraia novum genus de Triatomidae, Mepraia spinolai (Porter, 1933), nov. comb. redescripción del macho y descripcion de Ia hembra. Misión de Estudios de Patología Regional Argentina, Publicación 44: 1-27.

Schaub, G. A. 1998. Trypanosoma cruzi in the rectum of the bug Triatoma infestans: Effects of blood ingestion by the starved vector. Am. J. Trop. Med. Hyg. 59: 166-170.
Schaub, G. A., and C. A. Böker. 1986. Colonization of the rectum of Triatoma infestans by Trypanosoma cruzi: Influence of starvation studied by scanning electron microscopy. Acta Trop. 43: 349-354.

Schijman, A. G., M. Isio, L. Orellana, M. Sued, M. T. Duffy, A. M. Mejia Jaramillo, C. Cura, F. Auter, V. Veron, Y. Qvarnstrom, et al. 2011. International study to evaluate PCR methods for detection of Trypanosoma cruzi DNA in blood samples from Chagas disease patients. PLoS Negl. Trop. Dis. 5: e931. 2011 Jan 11doi: 10.1371/journal.pntd.0000931.

Vargas, L. G., and R. Zeledón. 1985. Effect of fasting on Trypanosoma cruzi infection in Triatoma dimidiata (Hemiptera: Reduviidae). J. Med. Entomol. 22: 683. 